\title{
Coronal loop oscillations and diagnostics with Hinode/EIS
}

\author{
Y. Taroyan ${ }^{1}$ and S. Bradshaw ${ }^{2}$ \\ ${ }^{1} \mathrm{SP}^{2} \mathrm{RC}$, Department of Applied Mathematics, University of Sheffield, Sheffield S3 7RH, UK \\ e-mail: y.taroyan@sheffield.ac.uk \\ 2 Space \& Atmospheric Physics, Blackett Laboratory, Imperial College London, Prince Consort Road, London SW7 2BZ, UK \\ e-mail: s.bradshaw@imperial.ac.uk
}

Received 4 September 2007 / Accepted 17 January 2008

\begin{abstract}
Context. Standing slow (acoustic) waves commonly observed in hot coronal loops offer a unique opportunity to understand the properties of the coronal plasma. The lack of evidence for similar oscillations in cooler loops is still a puzzle.

Aims. The high cadence EIS instrument on board recently launched Hinode has the capability to detect wave motion in EUV lines both in the imaging and spectroscopy modes. The paper aims to establish the distinct characteristics of standing and propagating acoustic waves and to predict their footprints in EIS data.

Methods. A 1D hydrodynamic loop model is used and the consequences of various types of heating pulses are examined. In each case, the resulting hydrodynamic evolution of the loop is converted into observables using a selection of available EIS spectral lines and windows.

Results. Propagating/standing acoustic waves are a natural response of the loop plasma to impulsive heating. Synthetic EIS observations of such waves are presented both in the imaging and spectroscopy modes. The waves are best seen and identified in spectroscopy mode observations. It is shown that the intensity oscillations, unlike the Doppler shift oscillations, continuously suffer phase shifts due to heating and cooling of the plasma. It is therefore important to beware of this effect when interpreting the nature of the observed waves.
\end{abstract}

Key words. Sun: atmosphere - Sun: oscillations - waves - hydrodynamics - line: profiles

\section{Introduction}

The problem of solar coronal heating first became apparent more than six decades ago. Since then a number of heating theories have been put forward none of which has yet been confirmed. Some of these theories merely represent ideas and concepts. Others are much better developed and involve sophisticated analytical and numerical modeling. Solving the coronal heating problem consists of a number of steps which involve their unique problems and challenges. The relationship between these steps is very important. It is easy to forget about the big picture while one is trying to focus on certain isolated aspects of the problem (Klimchuk 2006). A two-way interaction between theories and observations is an important and integral part of solving the heating problem. Such an interaction can only be provided by well developed forward modeling and inversion.

All of the proposed mechanisms require a coupled treatment of dynamically evolving small and large spatial scales which currently poses a severe challenge for multidimensional numerical modeling. Small scales such as current sheets and resonant surfaces are on the order of meters, whereas large scales such as magnetic loop structures are on the order of mega-meters. As a consequence, the treatment often relies on rather idealized modeling. Nevertheless there is still much to be learned even from simple models. A good example are the results of forward modeling in 1D (see, e.g., Mariska 1988; Cargill 1993; Hansteen \& Wikstol 1994; Reale et al. 1996; Patsourakos \& Klimchuk 2006). Using a 1D model and assuming that the heating process is impulsive, Taroyan et al. (2006) were able to qualitatively reconstruct the average red shifts commonly observed in transition region lines (see, e.g., Peter \& Judge 1999). In relation to this problem see also Hansteen (1993).

It is well known that the spectral, spatial and temporal resolution of current space-borne and ground based instruments is limited and this has been a major obstacle for development. Another inhibiting factor which has been somewhat overlooked by theoreticians is the lack of reliable inversion methods. Among the well-known methods for determining temperature and density profiles along the loops are those based on the analysis of intensity ratios which are derived using either imagers or spectrometers. The use of such methods has recently been questioned because they have often led to contradictory results (see, e.g., Landi \& Landini 2005; Schmelz \& Martens 2006). An example illustrating the existing controversy is a single dataset interpreted in terms of uniform (Priest et al. 1998), footpoint (Aschwanden 2001) and apex (Reale 2002) heating.

MHD waves offer new opportunities for plasma diagnostics. Taroyan et al. (2007a) applied a forward modeling approach to confirm the nature of the standing waves seen by SoHO/SUMER, the triggering mechanism and the energies involved. Interestingly, standing acoustic type waves have so far been detected only in hot $(T>6 \mathrm{MK})$ loops. The reason why such oscillations are not seen in cooler loops is still a puzzle. The EIS imaging spectrometer on board the new Hinode satellite could perhaps offer clues due to its high cadence, improved spatial resolution and feature tracking capability. One of the main aims of the present followup paper is to predict the footprints of standing and propagating waves in EIS observations. The results are presented both in imaging mode and in spectroscopy mode synthetic observations. 
The presence of individual coherent MHD waves is not guaranteed. Recently Taroyan et al. (2007b) have proposed a new inversion method which is based on the analysis of power spectra for Doppler shift time series. The heating takes place by short-lived pulses which are randomly distributed along the loop. The method could be used to distinguish uniformly heated loops from loops heated at their footpoints and has the potential to determine other unique footprints of the actual heating mechanism. The present paper also questions the applicability of the method to intensity times series.

\section{1D loop modeling}

In this section the one dimensional loop model is introduced and briefly reviewed. The use of $1 \mathrm{D}$ loop models is justified by the fact that the plasma and magnetic field are frozen together and the cross-field thermal conduction is greatly inhibited. The magnetic field plays only a passive role by channeling the plasma and thermal energy along the field lines. The advantage of using a 1D model is that highly complex field aligned behavior can be accurately simulated using a full energy equation. The disadvantage is that the heating must be specified and cannot be computed self-consistently (Klimchuk 2006).

\subsection{The governing equations}

The plasma motion along a loop is governed by the following set of nonlinear differential equations:

$\frac{\partial \rho}{\partial t}+\frac{\partial}{\partial s}[\rho v]=0$

$\frac{\partial}{\partial t}[\rho v]+\frac{\partial}{\partial s}\left[\rho v^{2}\right]=-\frac{\partial p}{\partial s}+\rho g_{\|}$

$\frac{\partial e}{\partial t}+\frac{\partial}{\partial s}[(e+p) v]=\rho v g_{\|}+\mathcal{H}-\frac{\partial F_{\mathrm{c}}}{\partial s}-\mathcal{L}$,

where $s$ is the coordinate along the loop, $\rho$ is density, $p$ is pressure, $v$ is velocity,

$e=\frac{p}{\gamma-1}+\frac{\rho v^{2}}{2}$.

is the energy density,

$g_{\|}=-g_{\odot} \cos \left(\frac{\pi s}{L}\right)$

is the component of gravitational acceleration along a semicircular loop of length $L$. The right-hand side of the energy equation contains sources and sinks of energy. Any type of heating term $\mathcal{H}$ must balance the combined thermal and radiative losses. The main losses in the corona. Thermal conduction is expressed in terms of the conductive flux $F_{\mathrm{c}}$ :

$F_{\mathrm{c}}=-\varkappa T^{5 / 2} \frac{\partial T}{\partial s}$

with $x=10^{-6} \mathrm{erg} \mathrm{s}^{-1} \mathrm{~K}^{-1} \mathrm{~cm}^{-1}$ being the coefficient of thermal conduction along the magnetic field. The gradient of conductive flux, i.e., thermal conduction can have both positive and negative values along the loop. It becomes positive in the lower transition region to balance losses due to strong radiation. The last term $\mathcal{L}=n^{2} \Lambda(T)$ corresponds to optically thin radiative losses where $n$ is the number density and $\Lambda=\Lambda(T)$ is the radiative loss function. The radiative losses are obtained from a table of values calculated using version 5 of the Chianti atomic database (Dere et al. 1997; Landi et al. 2006), tabulated as a function of temperature and density. Thermal bremmstrahlung is also included in the radiation calculation. The ionization balance is assumed to be in equilibrium and has been calculated from the ionization and recombination rates provided by Mazzotta et al. (1998). The element abundances are those due to Feldman (1992).

The chromosphere has an initial depth of $1.5 \mathrm{Mm}$ at each end of the loop and a uniform temperature of $T_{\text {ch }}=20000 \mathrm{~K}$. The chromospheric temperature is maintained against radiative losses using the method described by Klimchuk et al. (1987), where the radiative losses are smoothly decreased to zero over a small temperature interval, $\mathrm{d} T$ (100 $\mathrm{K}$ in the present work), where $T_{\text {ch }} \leq T \leq T_{\text {ch }}+\mathrm{d} T$. This has the effect of maintaining chromospheric stability such that it may act as a source/sink of mass and energy, while avoiding any significant artificial steepening of the radiative loss function at lower temperatures. The boundary conditions are such that the temperature is fixed at $20000 \mathrm{~K}$ and the bulk flow velocity is zero at the edges of the computational domain. We find that the chromosphere in our model is sufficient to damp any significant perturbations that may arise, long before they reach the edges of the domain.

\subsection{The role of the transition region}

Not including the transition region in the model could result in unphysical coronal solutions to the governing Eq. (1) in the sense that there does not exist a matching solution in the transition region. Further, thermal conduction transfers a large part of the energy deposited in the corona down to the transition region where it is more efficiently radiated due to higher densities and lower temperatures. The transition region is therefore very important for coronal diagnostics and it must be included in the model.

In the subsequent hydrodynamic simulations the heating rate is impulsive, i.e., $\mathcal{H}=\mathcal{H}(t, s)$ depends on both time and distance. The transition region moves up and down in response to changing pressure. Not resolving this region properly could produce significant errors in the coronal quantities. It is clear that an adequate treatment of the hydrodynamic evolution of the loop requires an adaptive mesh. In simple terms, points are dynamically added in places where they are needed and removed in places where they are no longer necessary. These features are implemented in HYDRAD (Bradshaw \& Mason 2003) which is used to integrate the governing Eq. (1).

\section{Results}

A $30 \mathrm{Mm}$ long loop with an apex temperature of around $1 \mathrm{MK}$ is chosen for the analysis. For simplicity, it is assumed that it has a semicircular shape and no inclination with respect to the vertical plane. The footpoints are anchored in a cool dense chromosphere. The loop is initially in hydrostatic equilibrium and a uniform heating of $\mathcal{H}_{0}=9 \times 10^{-4} \mathrm{erg} \mathrm{cm}^{-3} \mathrm{~s}^{-1}$ is applied to balance the losses. The analytical form of the pulse which leads to the formation of a standing wave is given by Taroyan et al. (2005):

$h= \begin{cases}h_{0} \sin ^{2}\left(\frac{\pi t}{P}\right) \exp \left(-\frac{\left|s-s_{0}\right|}{s_{h}}\right), & 0 \leq t \leq P, \\ 0, & t>P .\end{cases}$

Such a pulse with a scale length of $s_{h}=2 \mathrm{Mm}$ is applied at the top of the chromospheric footpoint of the present loop at 

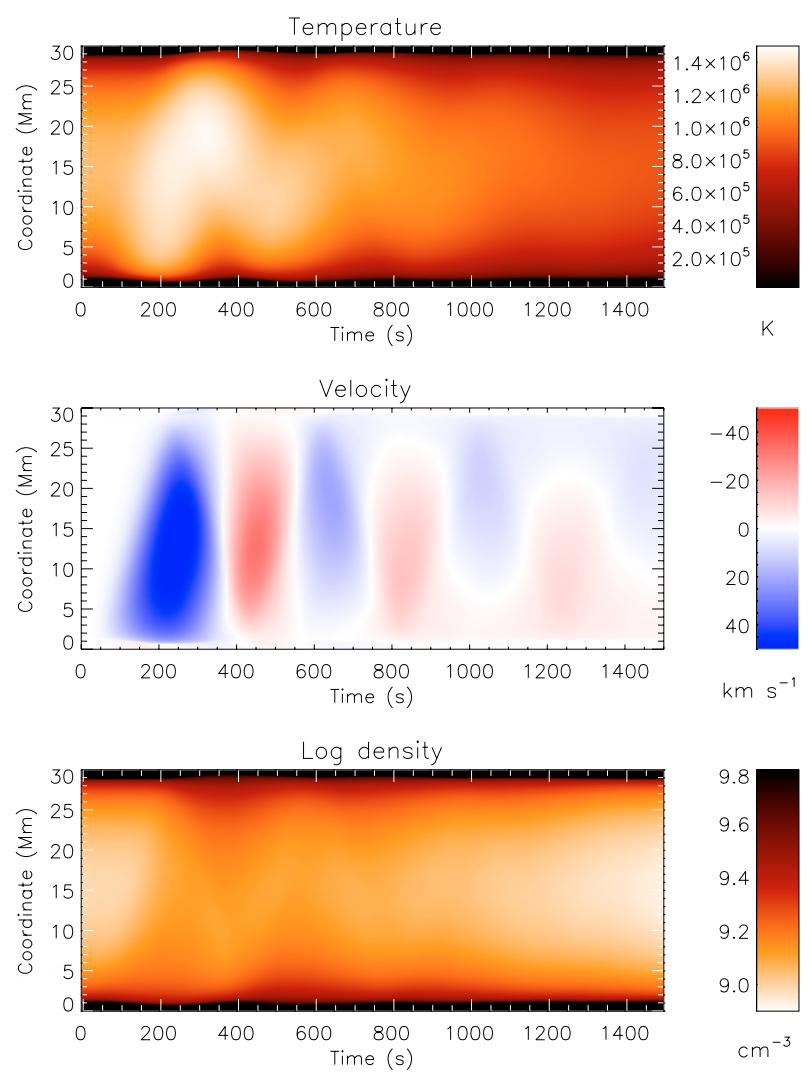

Fig. 1. The hydrodynamic evolution of the loop following a heating pulse near the lower footpoint between $0 \mathrm{~s}<t<350 \mathrm{~s}$.

$s_{0}=1.5 \mathrm{Mm}$. The main requirement for the formation of a standing wave is that the duration of the pulse $P$ should be approximately equal to the period of the fundamental mode.

The evolution of temperature, velocity and density along the loop is displayed in Fig. 1 for the first 1500 s. The impulsive heat deposition increases the temperature of the loop. The pulse has a maximum rate of $h_{0}=2.5 \times 10^{-2} \mathrm{erg} \mathrm{cm}^{-3} \mathrm{~s}^{-1}$ and lasts between $0 \mathrm{~s}$ and $350 \mathrm{~s}$. The maximum heat flux and total energy input into the loop are $7.5 \times 10^{6} \mathrm{erg} \mathrm{cm}^{-2} \mathrm{~s}^{-1}$ and $9.4 \times 10^{8} \mathrm{erg} \mathrm{cm}^{-2}$, respectively. As the transient heating is over, the loop begins to cool due to the combined action of thermal conduction and radiation. The fundamental mode period is determined by the ratio of the loop length and the sound speed which is proportional to the square root of temperature. The loop length remains constant and therefore the wave period varies because of heating and cooling, i.e., changes in temperature. The time distance diagram for the velocity shows a standing wave pattern which gradually gets deformed as the loop cools to lower temperatures and the oscillation no longer represents an eigenmode of the system. The velocity oscillation is rapidly damped. The density suffers a temporary increase following the injection of heat at the footpoint. Figure 2 displays the hydrodynamic evolution of the loop when a pulse with a shorter duration is applied at the left footpoint. The heating lasts between $0 \mathrm{~s}<t<70 \mathrm{~s}$ and has a maximum rate of $h_{0}=5 \times 10^{-2} \mathrm{erg} \mathrm{cm}^{-3} \mathrm{~s}^{-1}$. The corresponding maximum heat flux and total energy input into the loop are $1.5 \times 10^{7} \mathrm{erg} \mathrm{cm}^{-2} \mathrm{~s}^{-1}$ and $5.2 \times 10^{8} \mathrm{erg} \mathrm{cm}^{-2}$, respectively. The time distance plot for the velocity shows that the pulse propagates back and forth inside the loop. There are no standing wave patterns like in Fig. 1. The total energy injected at the footpoint is smaller compared to the previous case, so the loop cools and the motions vanish faster. Therefore only the first $950 \mathrm{~s}$ of the evolution are plotted.
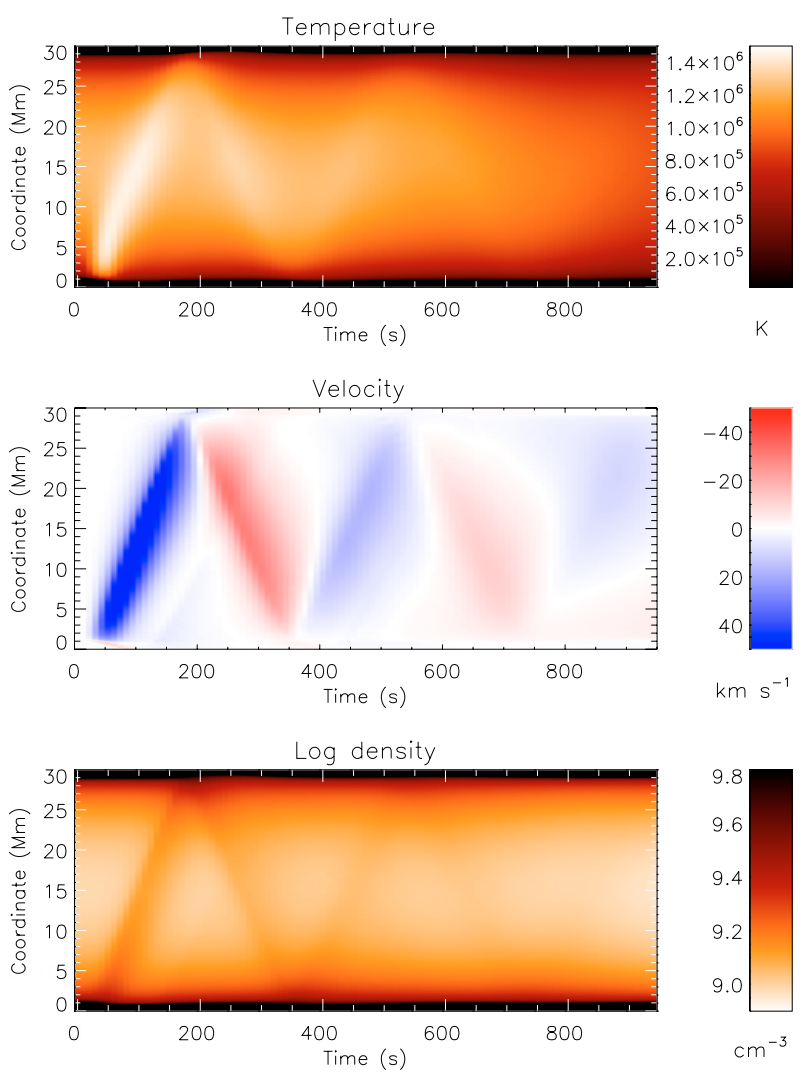

Fig. 2. The hydrodynamic evolution of the loop following a heating pulse near the lower footpoint between $0 \mathrm{~s}<t<70 \mathrm{~s}$.

In the next part of the section, the results of hydrodynamic simulations are converted and presented in terms of observable quantities. The details of the applied procedure are described by, e.g., Taroyan et al. (2006).

The EUV imaging spectrometer Hinode/EIS has two CCDs each covering a $40 \AA$ wavelength range: 170-210 $\AA$ and 170-210 A. The wavelength response of EIS has two peaks at around $195 \AA$ and $271 \AA$ corresponding to the two CCDs. The response function is used when synthesizing observables from the simulations. EIS has both narrow ( $1^{\prime \prime}$ and $2^{\prime \prime}$ wide) slits, and wider (40" and 266") imaging slots, all with 512" in the Solar Y direction. EIS should be able to make slit images of active regions in $10 \mathrm{~s}$, of quiet Sun in between 30 and $60 \mathrm{~s}$, and of flares in approximately one second. The spectral resolution can be less than $3 \mathrm{~km} \mathrm{~s}^{-1}$ for the Doppler shift. Further details of Hinode/EIS characteristics are given by Culhane et al. (2007), Kosugi et al. (2007).

In the present paper, the simulation results are converted and downgraded into EIS observables assuming an exposure time of $20 \mathrm{~s}$ and a spatial resolution of $1^{\prime \prime}$. The period is proportional to the loop length. Simple estimates show that for a temporal resolution of, e.g., $30 \mathrm{~s}$ lengths greater than $10 \mathrm{Mm}$ are required for the oscillations to be detected in loops with temperatures of around $1 \mathrm{MK}$. It is assumed that the observations are carried out in a sit-and-stare mode assuming and the loop remains in the field of view of EIS. Three iron lines representing different temperatures are selected: Fe XII $195 \AA$, Fe XI 188.23 $\AA$ and Fe X $190 \AA$. The $40^{\prime \prime}$ slot and the $1^{\prime \prime}$ slit are selected to represent the results in the imaging mode and in the spectroscopy mode, respectively. The heliographic position of the loop and its orientation must be specified. For simplicity, it is assumed that the 

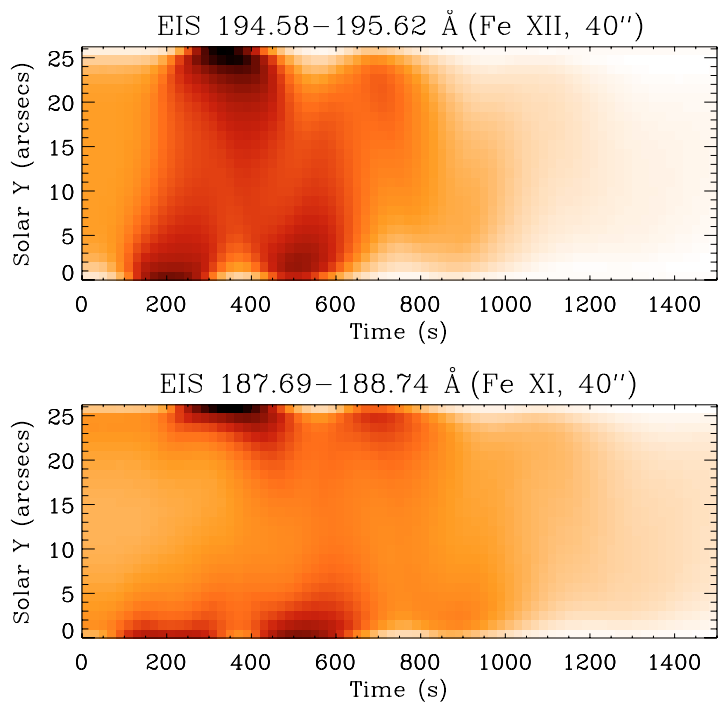

EIS $189.5-190.5 \AA\left(F e X, 40^{\prime \prime}\right)$

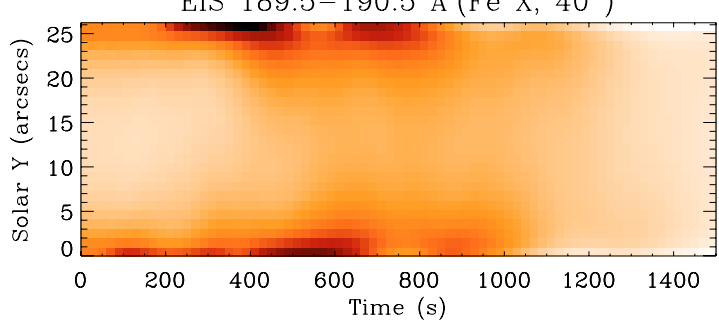

Fig. 3. Synthetic observations of a standing wave by Hinde/EIS in the imaging mode corresponding to Fig. 1. The results are presented in three different wavelengths with a $40^{\prime \prime}$ slot and exposure time of $20 \mathrm{~s}$.
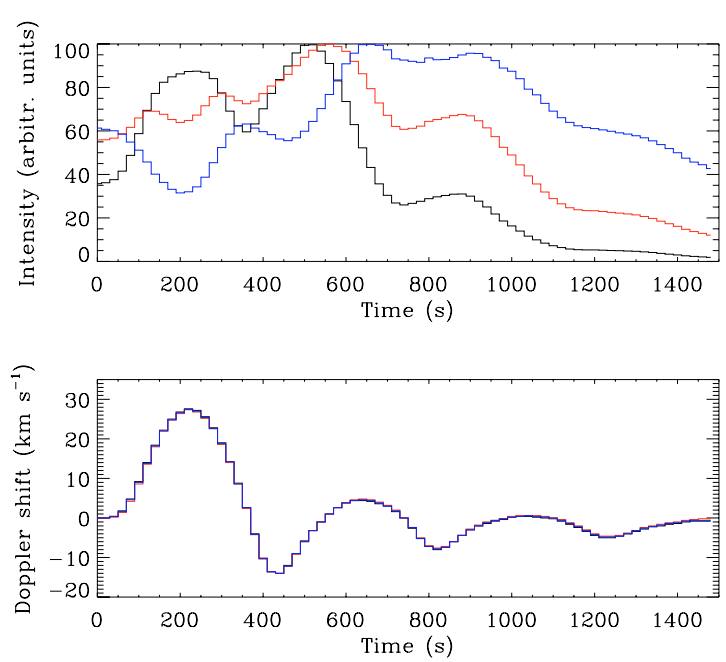

Fig. 4. Synthetic observations of a standing wave by Hinde/EIS in the spectroscopy mode corresponding to Fig. 1. Three different iron lines with a 1" slot are used. The black, red and blue lines correspond to Fe XII, Fe XI and Fe X, respectively.

loop is at the disc center and is oriented along the EIS slit in the south-north direction.

The results are first presented in the imaging mode. Figure 3 shows the synthesized emission in EIS with the 40" slot. All of the ions which may have a contribution to the emission in the given wavelength range are taken into account. The images are for the standing wave shown in Fig. 1. The coordinate in the
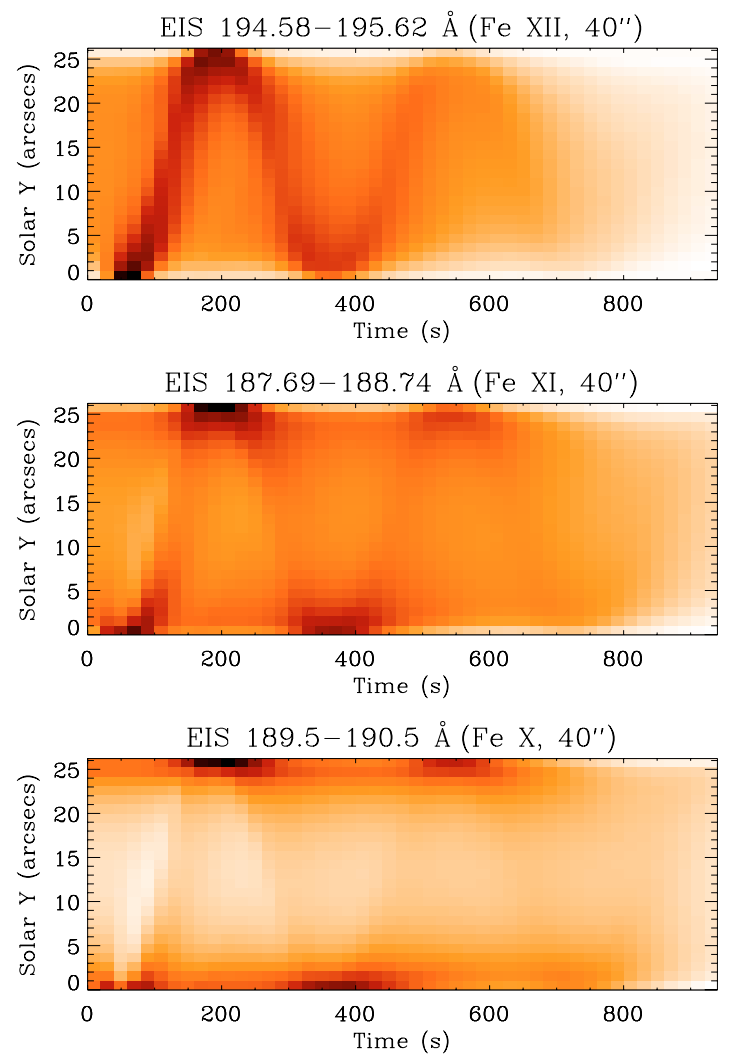

Fig. 5. Synthetic observations of a propagating wave by Hinde/EIS in the imaging mode corresponding to Fig. 2. The results are presented in three different wavelengths with a $40^{\prime \prime}$ slot and exposure time of $20 \mathrm{~s}$.
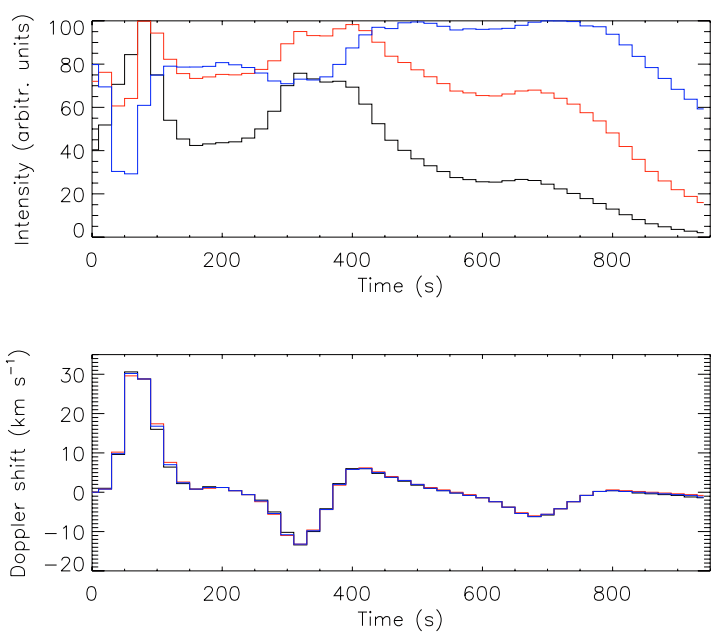

Fig. 6. Synthetic observations of a propagating wave by Hinde/EIS in the spectroscopy mode corresponding to Fig. 2. Three different iron lines with a $1^{\prime \prime}$ slot are used. The black, red and blue lines correspond to Fe XII, Fe XI and Fe X, respectively.

vertical direction is the projection of the loop coordinate $s$ on the plane normal to the line of sight:

$y=\frac{2 L}{\pi} \sin ^{2}\left(\frac{\pi s}{2 L}\right)$

Dark regions represent strong emission and bright regions correspond to weak emission. The event is most clearly seen in the top Fe XII window when the loop is heated to high 


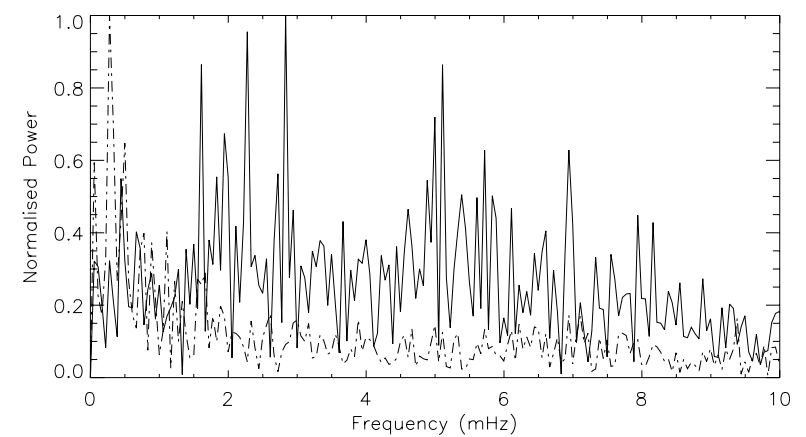

Fig. 7. Power spectra of the Doppler shift and intensity time series for the Fe $\mathrm{X}$ line. The solid and dash-dotted lines correspond to the Doppler shift and the intensity, respectively.

temperatures and the oscillation has just begun. The other two windows indicate an oscillatory behavior mainly near the footpoints of the loop. The results of the same hydrodynamic simulation are also presented in spectroscopic observations using the $1^{\prime \prime}$ slit in a sit-and-stare mode. The total intensity of a spectral line is integrated along a $4^{\prime \prime}$ slit cut from $s=10 \mathrm{Mm}$ down towards the lower footpoint. The choice allows us to study the oscillations both in the intensity and in the Doppler shift. The evolution of the intensity is plotted in the top panel of Fig. 4. Arbitrary units have been used by normalizing the intensity with respect to its maximum value. The corresponding Doppler shifts are plotted in the bottom panel. The black, red and blue lines represent Fe XII, Fe XI and Fe X, respectively. The initial plasma inflow leads to a positive blue shift which is followed by a damped oscillation. The intensity plots show oscillations superimposed on the background intensity trend. The spectroscopy mode observations indicate oscillatory behavior in all three lines. The quarter period phase shift between the intensity and Doppler shift oscillations seen, for example, in the Fe $\mathrm{X}$ during the initial stages of evolution is a well known characteristic of a standing wave (see, e.g., Taroyan et al. 2007a). Figure 4 shows that the phase of the intensity oscillations undergoes variations in all three lines as it passes through its maximum.

A similar procedure is applied to the propagating wave solution in Fig. 2 and the resulting synthetic EIS observations are presented in Fig. 5 for the imaging mode and in Fig. 6 for the spectroscopy mode. The slit/slot selection and the colors used to indicate different lines are the same as those used in Figs. 3 and 4 . It is instructive to compare the synthesized observations of a standing wave with those of a propagating wave. The imaging mode diagrams show that the emission evolves more smoothly in the case of a standing wave. The Doppler peaks are sharper in Fig. 6 compared to those in Fig. 4. The amount of energy required for setting up a standing wave (Fig. 4) is almost twice the amount of energy needed for the propagating wave (Fig. 6) with a similar amplitude. A comparison between the top and bottom panels of Figs. 4 and 6 shows that in both cases variations in the phase of the intensity oscillations is present. To further explore this phenomenon, we have simulated impulsive heating of the same loop by random pulses for $5 \mathrm{~h}$. The temperature near the loop apex varies between $0.9 \mathrm{MK}$ and $1.3 \mathrm{MK}$ with an average of about $1 \mathrm{MK}$. The intensity and Doppler shift time series for Fe X are Fourier analyzed and the resulting power spectra are plotted in Fig. 7. The solid and dash-dotted lines correspond to the Doppler shift and the intensity, respectively. The comparison between the two curves is striking. The power peaks for the Doppler shift time series are clustered around the frequencies of standing wave harmonics $(\omega \approx 2.5,5 \mathrm{mHz})$ as predicted by Taroyan et al. (2007b). These results are confirmed by the wavelet analysis (not shown). On the other hand, the only significant peak for the intensity time series is located at very low frequencies and is a consequence of the finite duration of the random pulses $(<10 \mathrm{~s})$. The peaks corresponding to the normal modes are absent because the intensity oscillations continuously suffer phase variations. A mathematical explanation can be given by representing the total intensity as a superposition of a monotonous background and an oscillation:

$I(t)=I_{0}(t)+I_{1} \cos \left(\frac{2 \pi t}{P}\right)$,

where $I_{1}$ is the amplitude of the oscillation and $P$ is the wave period. As time evolves, the background term $I_{0}(t)$ increases, reaches its peak at $t_{0}$ and decreases back. This could represent either cooling or heating of the plasma. Let $t_{-}\left\langle t_{0}\right.$ and $\left.t_{+}\right\rangle t_{0}$ be the positions of any two peaks for the total intensity $I(t)$. We have

$I_{0}^{\prime}\left(t_{-}\right)=\frac{2 \pi I_{1}}{P} \sin \left(\frac{2 \pi t_{-}}{P}\right)>0, \quad I_{0}^{\prime}\left(t_{+}\right)=\frac{2 \pi I_{1}}{P} \sin \left(\frac{2 \pi t_{+}}{P}\right)<0$.

Therefore, the time difference $\delta t=t_{+}-t_{-}$cannot be a multiple of the wave period $P$ : this would imply $I_{0}^{\prime}\left(t_{-}\right)=I_{0}^{\prime}\left(t_{+}\right)$whereas, according to Eq. (8), the signs of $I_{0}^{\prime}\left(t_{-}\right)$and $I_{0}^{\prime}\left(t_{+}\right)$are opposite. In other words, the total intensity oscillation undergoes a phase shift whenever the background intensity passes through its peak. In the particular case of $I_{0}^{\prime}\left(t_{-}\right)=-I_{0}^{\prime}\left(t_{+}\right)$, according to Eq. (8), the phase shift is equal to half a period. Such a behavior is seen, e.g., in the case of the Fe X line in Fig. 4.

\section{Discussion and conclusions}

Previous studies have mainly concentrated on standing acoustic waves in hot loops with temperatures in excess of $6 \mathrm{MK}$ (e.g., Wang et al. 2002, 2005). The main reason is that hot loops are the only ones in which these type of oscillations are currently being observed. These waves are usually set up rather quickly and decay within a few wave periods. The strong damping is mainly due to thermal conduction (Ofman \& Wang 2002). The oscillations are preceded by an inflow of hot plasma at one of the footpoints. This could be due to a reconnection process between neighboring loops although the exact origin of the inflow is not yet clear. Taroyan et al. (2005) found an exact analytical form for the pulse at the footpoint which sets up a standing wave inside the loop within a single period. Such a pulse was applied to an active region loop in which standing waves were detected (Taroyan et al. 2007a). The subsequent behavior of the loop was examined taking into account the loop inclination, heliographic position, temperature, gravitational acceleration and the effects of thermal conduction and radiation. A successful comparison between the observed and simulated loop behavior in several spectral lines allowed us to determine the exact nature of the observed waves, the energies involved in the pulse and the time distance profile of the heating function along the loop. It is not yet clear why these oscillations are only being seen in high temperature lines. Figure 1 and the velocity diagram, in particular, shows that standing waves can be formed in cooler EUV loops in a similar way when all the important effects such as gravitational and thermal stratification, losses, etc., are taken into account. Hot loops are therefore not unique in this respect and there is nothing to prevent the formation of standing waves in cooler loops. 
Both standing and propagating waves are a natural response of the loop plasma to impulsive heating. The results are presented in terms of synthetic EIS observations to predict the wave footprints in the actual observations. In the case of imaging mode observations, the waves are most clearly seen in the EIS Fe XII $195 \AA$ filter when they are just being set up. In contrast to this, the waves clearly appear in all three lines when spectroscopic observations are applied. The quarter period phase shift between the intensity and the Doppler shift oscillations is an indicator of a standing wave. It is shown that the intensity oscillations suffer phase variations when the plasma undergoes heating or cooling. A simple analytical model is used to mathematically explain this phenomenon. Individual coherent MHD waves are not very often seen. Taroyan et al. (2005b) have proposed a new diagnostic method which does not require the presence of such waves. It is based on the analysis of Doppler shift time series and is similar to the approach adopted in helioseismology. The results of the present paper show that this method cannot be successfully applied to the intensity time series because of the phase variations. However, the power spectrum of the Doppler shift time series is quite sensitive to the spatial and temporal distribution of the heating function (Taroyan et al. 2007b). The full potential of this new promising approach has yet to be explored.

Acknowledgements. Y.T. is grateful to the Leverhulme Trust for financial support. S.J.B. is grateful to PPARC for their support through the award of a Post-Doctoral Fellowship.

\section{References}

Aschwanden, M. J. 2001, A\&A, 560, 1035

Bradshaw, S. J., \& Mason, H. E. 2003, A\&A, 407, 1127

Cargill, P. J. 1993, Sol. Phys., 147, 263,

Culhane, J. L., Harra, L. K., James, A. M., et al. 2007, Sol. Phys., 243, 19

Dere, K. P., Landi, E., Mason, H. E., Monsignori, B. C., \& Young, P. R. 1997,

A\&AS, 125, 149

Feldman, U. 1992, Phys. Scr., 46, 202

Hansteen, V. H. 1993, ApJ, 402, 741

Hansteen, V. H., \& Wikstol, O. 1994, A\&A, 290, 995

Klimchuk, J. 2006, Sol. Phys., 234, 41

Klimchuk, J. A., Antiochos, S. K., \& Mariska, J. T. 1987, ApJ, 320, 409

Kosugi, T., Matsuzaki, K., Sakao, T., et al. 2007, Sol. Phys., 243, 3

Landi, E., \& Landini, M. 2005, ApJ, 618, 1039

Landi, E., Del Zanna, G., Young, P. R., et al. 2006, ApJS, 162, 261

Mariska, J. T. 1988, ApJ, 334, 489

Mazzotta, P., Mazzitelli, G., Colafrancesco, S., \& Vittorio, N. 1998, A\&AS, 133,403

Ofman, L., \& Wang, T. J. 2002, ApJ, 580, L85

Patsourakos, S., \& Klimchuk, J. 2006, ApJ, 647, 1452

Peter, H., \& Judge, P. G. 1999, ApJ, 522, 1148

Reale, F. 2002, ApJ, 580, 566

Reale, F., Peres G., \& Serio S. 1996, A\&A, 316, 215

Priest, E. R., Foley, C. R., Heyvaerts, J., et al. 1998, Nature, 393, 545

Schmelz, J. T., \& Martens, P. C. H. 2006, ApJ, 636, L49

Taroyan, Y., Erdélyi, R., Bradshaw, S. J., \& Doyle, J. G. 2005, A\&A, 438, 713

Taroyan, Y., Bradshaw, S. J., \& Doyle, J. G. 2006, A\&A, 446, 315

Taroyan, Y., Erdélyi, R., Wang, T. J., \& Bradshaw, S. J. 2007a, ApJ, 659, L173

Taroyan, Y., Erdélyi, R., Doyle, J. G., \& Bradshaw, S. J. 2007b, A\&A, 462, 331

Wang, T. J., Solanki, S. K., Curdt, W., Innes, D. E., \& Dammasch, I. E. 2002,

ApJ, 574, L101

Wang, T. J., Solanki, S. K., Innes, D. E., \& Curdt, W. 2005, A\&A, 435, 753 